\title{
Implementation of a 2D Electrostatic Particle in Cell algorithm in Unified Parallel C with dynamic load-balancing
}

\author{
B. Verleye ${ }^{*, 1,4}$, P. Henri ${ }^{2,4}$, R. Wuyts ${ }^{3,4}$, G. Lapenta ${ }^{2}$, K. Meerbergen ${ }^{1}$ \\ ${ }^{*}$ Corresponding author: bart.verleye@cs.kuleuven.be \\ ${ }^{1}$ K.U.Leuven, Computer Science, Celestijnenlaan 200A, B-3001 Leuven, Belgium \\ ${ }^{2}$ K.U.Leuven, Centre for Plasma Astrophysics, Celestijnenlaan 200B, B-3001 Leuven, Belgium \\ ${ }^{3}$ IMEC, Kapeldreef 75, B-3001 Leuven, Belgium \\ ${ }^{4}$ Intel ExaScience Lab Leuven, Kapeldreef 75, B-3001 Leuven, Belgium
}

\begin{abstract}
Since space plasmas are colisionless, reliable space weather simulations will require to take into account a full kinetic description of their dynamics. The Particle in Cell (PIC) method is a particle-mesh technique that efficiently allows such description. Still, realistic plasma simulations require huge data sets and are computationally expensive. High scalability must thus be achieved in order to perform the massively parallel plasma simulations required for space weather purposes. This paper reports on the first implementation of a 2D electrostatic PIC code in Unified Parallel C which also includes load balancing capabilities.
\end{abstract}

Keywords: PIC, particle-mesh techniques, UPC, plasma physics, space weather, CFD, Load-Balancing

\section{Introduction}

Most laboratory, space and astrophysical plasmas are collisionless. Their dynamics is described by the Vlasov equation self consistently coupled to Maxwell's equation [9]. When applied to space weather simulations, such a system of equations requires too huge data sets and is computationally expensive. Current HPCs are not sufficiently large to compute desired data. Practically, the Vlasov-Maxwell system is thus commonly solved using a particle-mesh method, the so-called Particle-in-Cell (hereafter PIC) method $[1,6]$. We hereafter concentrate on the electrostatic case, i.e. when the magnetic field is neglected, for which the Vlasov equation is self-consistently coupled with only the Poisson equation.

It has recently been shown, in the case of a 1D implementation, that the Unified Parallel C (UPC) language can be used efficiently for the development of PIC codes [10], and may be promising for scalability purposes. This paper reports the first implementation of a $2 \mathrm{D}$ electrostatic PIC code in UPC. The code also includes load balancing capabilities in order to improve its scalability.

In the PIC method, individual macro-particles in a Lagrangian frame which mimic the behavior of the distribution function are tracked in continuous phase space. Moments of the distribution function such as densities and currents are computed simultaneously on an Eulerian 
frame (fixed cells). The particle in cell method has four main steps that are executed in every time step:

(i) The charge density is interpolated on the grid from the particles position $\left(x_{p} \rightarrow \rho_{g}\right)$. We here use a cloud-in-cell scheme.

(ii) The Poisson equation is solved to compute the electric field on the grid $\vec{E}_{g}$ from the charge density $\rho_{g}$. $\left(\rho_{g} \rightarrow E_{g}\right)$.

(iii) The electric field is interpolated from the grid to the particle positions $\left(E_{g} \rightarrow E_{p}\right)$. The same interpolation as step (i) is used.

(iv) The particle velocities and positions are advanced in time with Newton's equations knowing the electric field at the particle positions: $d \vec{v}_{p} / d t=q_{s} / m_{s} \vec{E}_{p}$ and $d \vec{x}_{p} / d t=\vec{v}_{p}\left(E_{p} \rightarrow x_{p}\right)$.

Load imbalance often occur in parallel PIC simulations because particles are pushed on the initially balanced grid. Indeed, computational particle densities can vary by order of magnitude in time and space. Since particles (step (i) and (iv)) typically account for $90 \%$ of the computational time, these computational particle density inhomogeneities can have serious impacts on the code's scalability. This is why some dynamic load balancing is needed $[7,8]$.

\section{PIC data structures in UPC}

Unified parallel C is a Partitioned Global Address Space (PGAS) programming language [2, 4]. Briefly, it means that every executed thread can access shared data directly (global address), however, the data is partitioned and every thread keeps a part of the data local. Data that is not declared as shared is private and local, and only the owning thread can access the data. All threads can always read/write data that is declared shared: UPC applies the one-sided communication paradigm.

For the implementation of a PIC algorithm in UPC, two structures are required: a representation of the grid on which the solution of the potential is computed, and a structure that contains the particles, i.e. their position and velocity.

In the presented implementation, the grids are a pointer to an array of arrays. The pointer itself is private, however, equal for all threads. The arrays are shared. Figure 2 shows the array of pointers to the arrays. All data is accessible by each thread. The colours show the partitioning of the arrays among the threads.

In (UP)C, the particles are represented as a struct, with four doubles (position and velocity in two directions). Every thread has a local, but shared, array of particles. The particles in an array have a position that lies in the space determined by the grid points with affinity to the according thread. If particles are moved, they can also move from one array to the other. Therefore, the array of particles is made dynamic in size.

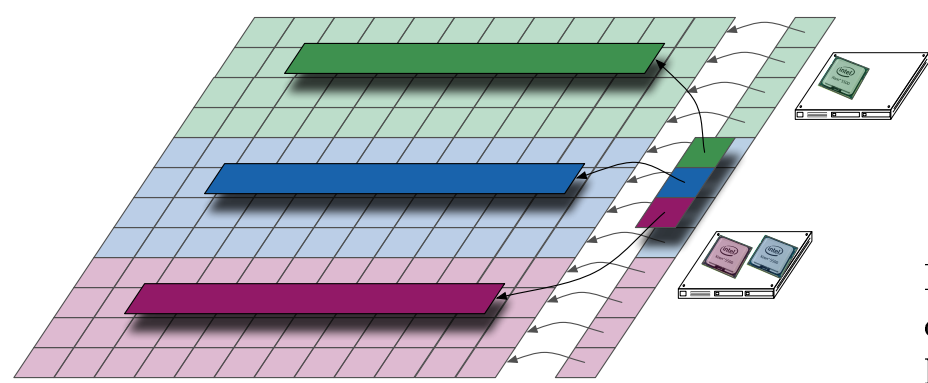

Figure 1: Representation of the PIC data structures in UPC. Every thread has affinity to several arrays of the grid, and to one array of particles. 


\section{The simulation algorithm in UPC}

There are several possible bottlenecks in the PIC algorithm when computed parallel. Depending on the number of particles, the main bottleneck is the potential solver or the update of the particles' position. For the field solver, a Conjugate Gradient (CG) solver [5] was chosen. As periodic boundary conditions are applied, one extra equation is added that fixes the solution, without losing symmetry [3].

The current implementation applies load-balancing in one direction. When one thread has too much work, or is working too slowly, rows and according particles are pushed to the neighbouring left thread. The measure for work in this case is the time it takes to update the velocity of all particles with affinity to the thread. The amount of work that is pushed, depends on the difference of work to the average work taken by all threads. To avoid too much communication cost, rows and particles are only pushed if the difference is significant.

\section{Numerical results}

\subsection{Physical test: two-stream instability}

To validate the PIC code, different tests have been done. The conservation of charge and momentum have been checked. The code is also tested for the two-stream instability, a classical collisionless plasma instability [9]. Particle positions are initialized with constant spacing in both directions so that the electron density is initially constant with $\rho=1$. A constant neutralizing background is added. The charge to mass ratio is $q_{s} / m_{s}=-1$ so that the plasma frequency is $\omega_{p}=1$. Particle velocities are chosen so that an equal number of particles start with velocities $V_{b}= \pm 0.05$. The time step $d t=0.1$ and grid spacing $d x=9.06910^{-3}$ are chosen to satisfy both stability conditions on the finite-grid instability and the leap-frog scheme.

We check that the fastest growing mode is the one given by the linear analysis of the cold twostream instability $k_{0}=\frac{\sqrt{3}}{2} \frac{\omega_{p}}{v_{b}}$, corresponding to a wavenumber $\mathrm{N}=4$ in the reported simulation. The time evolution of the Fourier transform of the electric field at wavenumber $k_{0}$ is shown in Figure 3 (red line). During the linear phase of the instability $\left(10<t \omega_{p}<30\right)$, the growth rate is equal to the theoretical one $\gamma_{0}=\frac{\omega_{p}}{2}$ [1], represented by the slope of the blue line.

The evolution of the particles in the phase space $\left(x, v_{x}\right)$ is shown in Figure 2. The initial
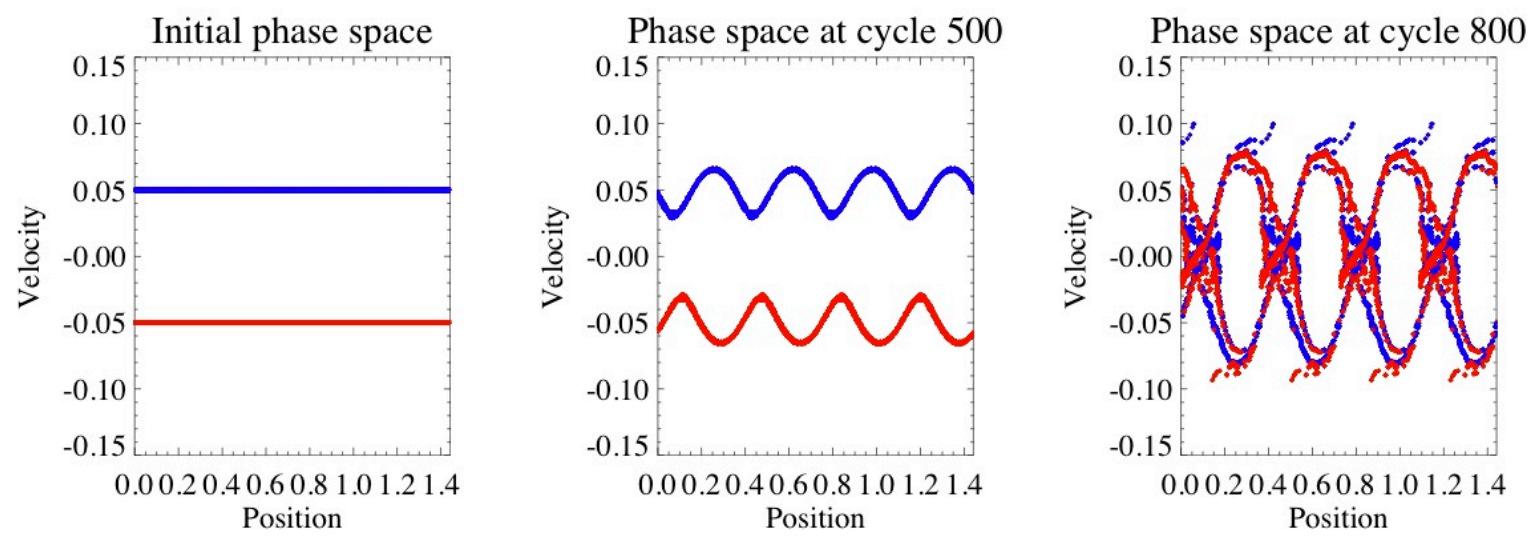

Figure 2: Particle evolution in the phase space $\left(x-v_{x}\right.$ space). Particles from the two initial beams are shown with different colors. 


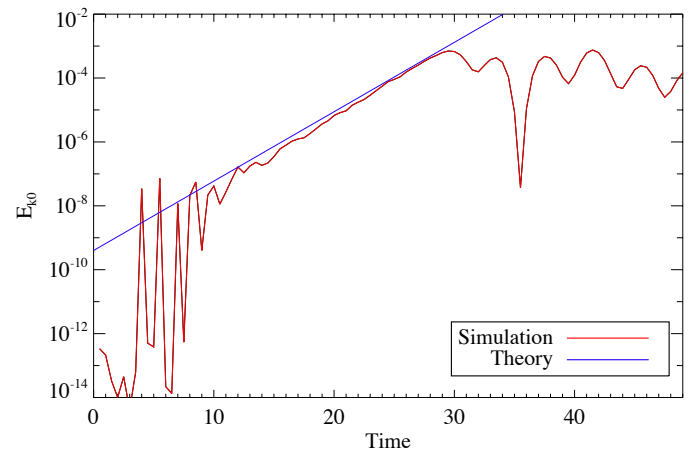

Figure 3: Evolution of the fastest growing mode $E_{k_{0}}$ during the simulation (red line) and slope of this expected theoretical growth rate for the linear phase of the instability (blue line). Time is normalized to $\omega_{p}^{-1}$.

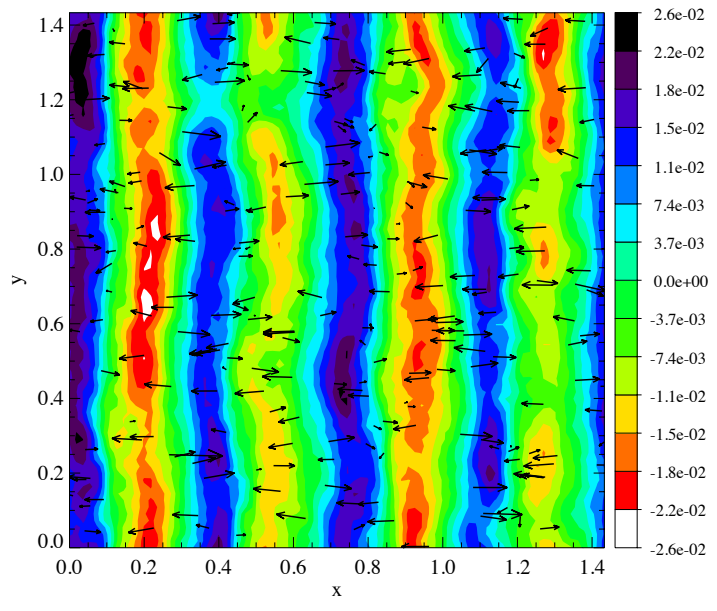

Figure 4: Snapshot of the electric field $E_{x}$ in the 2D simulation box, together with position and velocities (black arrows) of a sample of computational particles.

velocity beams (top panel) get unstable to the two-stream instability (linear phase shown in the medium panel at $t=25 \omega_{p}^{-1}$ ) and finally generate phase space vortices at saturation (bottom panel, $t=45 \omega_{p}^{-1}$ ). The electric field perturbation is initially generated along the $\mathrm{x}$-direction only. It then exhibits a $2 \mathrm{D}$ behavior during the saturation part of the instability because of wave-particle interactions (particle trapping). This feature is shown in Figure 4, where the contour plot shows $E_{x}$ in the 2D simulation box and the arrows shows the positions (origin of the arrows) and velocities (length of the arrow) of random computational particles.

\subsection{Load balancing and scaling}

The two-stream instability problem as described above was used for the scaling tests. For the weak scaling test, the problem size was $32^{*} \mathrm{Tx} 32$, with $\mathrm{T}$ the number of threads, and $2 \times 20 \times 20$ particles per cell. Figure 6 shows that the parallel program scales well. There is no optimal scaling, as the larger the problem, the more iterations the CG solver needs to converge, and the harder global communication gets. The table in Figure 5 presents the results of a strong scaling test, for a 5120x26 grid with $2 \times 15 \times 15$ particles per thread.

As the two-stream simulation keeps the particles almost homogeneously distributed, it is not a suitable test for dynamic load-balancing. Therefore, the load-balancing is tested with following initialisation: The initial position and velocity of the particles is given a sin-distribution, in such that the particles will flock together and separate every cycle of 30 time steps. The load-balancer reduces the computation time of step (i) and (iv) of the PIC algorithm. However, the combination of all timings and overhead avoiding strategies is indispensable to optimize the total computation time.

\section{Conclusion and future research}

This paper presents the implementation of a 2D electrostatic PIC code in UPC. The UPC language allows for a efficient design and implementation of the required data-structures. Results are presented for a two-stream instability simulation, which shows good strong and weak scaling. 


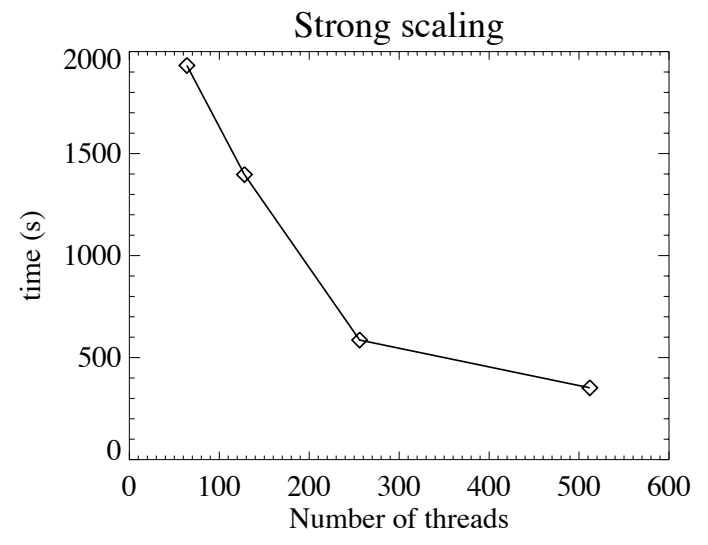

Figure 5: Strong scaling results for the twostream instability problem with 225 particles per cell and $n x \times$ ny $=5120 \times 26$ and $\mathrm{i}=100$.

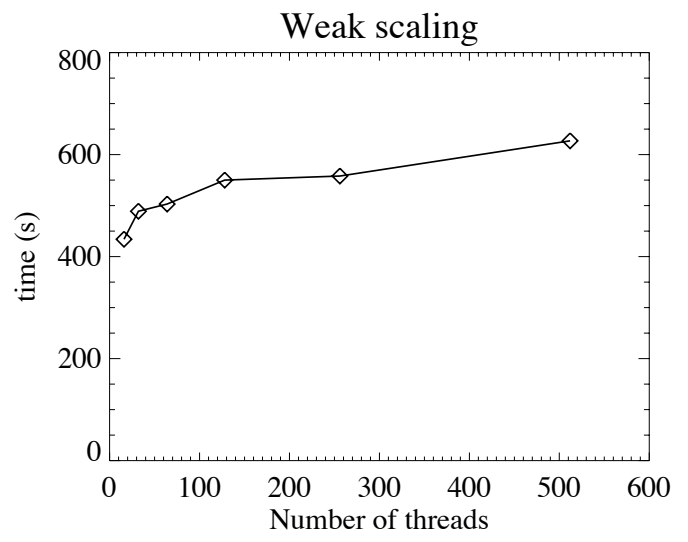

Figure 6: Weak scaling results for the twostream instability problem with 400 particles per cell and 32x32 grid cells per thread.

However, a straightforward CG solver will not scale up until thousands of threads. Therefore, the ExaScience Lab is working on communication avoiding strategies, also for other Krylov methods than the CG. Further research will also include the search for optimal load balancing strategies for PIC simulations.

Acknowledgements The authors acknowledge useful discussions with Stefano Markidis on PIC codes. This work is funded by the Institute for the Promotion of Innovation through Science and Technology in Flanders (IWT), and by Intel.

\section{References}

[1] C. K. Birdsall and A. B. Langdon. Plasma Physics via Computer Simulation. McGraw-Hill, New York, USA, 1985.

[2] W. Carlson, J. Draper, D. Culler, K. Yelick, E. Brooks, and K. Warren. Introduction to UPC and Language Specification, CCS-TR-99-157, IDA Center for Computing Sciences, 1999.

[3] A.R. Champneys, B. Sandstede. Numerical Computation of Coherent Structures, in Numerical Continuation Methods for Dynamical Systems, ed: B. Krauskopf et al., Springer Netherlands, 2007.

[4] T. El-Ghazawi, W. Carlson, T. Sterling, and K. Yelick. UPC: Distributed Shared Memory Programming, John Wiley \& Sons Inc., New York, 2005.

[5] M.R. Hestenes, E. Stiefel. Methods of Conjugate Gradients for Solving Linear Systems, Journal of Research of the National Bureau of Standards 49 (6), 1952.

[6] R. Hockney and J. Eastwood. Computer Simulation Using Particles. Taylor \& Francis, 1988.

[7] R. D. Ferraro, P. C. Liewer and V. K. Decyk. Dynamic Load Balancing for a $2 D$ Concurrent Plasma PIC Code, Journal of Computational Physics, 109, 329-341, 1993.

[8] S. J. Plimpton et al. A Load-Balancing Algorithm for a Parallel Electromagnetic Particle-in-Cell Code, Comp. Phys. Comm., 152, 227-241, 2003

[9] N. A. Krall and A. W. Trivelpiece. Principles of plasma physics. International Series in Pure and Applied Physics, McGraw-Hill, 1973.

[10] S. Markidis and G. Lapenta. Development and performance analysis of a UPC Particle-in-Cell code. 4th Conf. on Partitioned Global Address Space Programming Model (PGAS10), 2010. Numerical computation of coherent structures 